\title{
Numerical study of a turbulent lobed jet with variable density
}

\author{
Mohamed Boulenouar ${ }^{a}$ And Bachir Imine \\ Laboratoire d'aéronautique et des systèmes propulsifs, Faculté de Génie mécanique, Université Mohamed Boudiaf, Oran, Algérie
}

Received 8 July 2010, Accepted 14 November 2011

\begin{abstract}
Numerical simulations of the isothermal turbulent jets mixing flows exhausted from two different circular lobed nozzles are presented in the present paper. The numerical studies have been conducted using a Favre-Reynolds Averaged Navier-Stokes approach, using the second-order Reynolds Stress Model $(R S M)$ and structured mesh. The validation of the numerical results with experimental data has been carried out only with one nozzle for the same configuration. This comparison shows reasonable agreement, principally, in terms of centreline longitudinal velocity, longitudinal fluctuating velocity and streamwise vorticity. In the second part, the effects of the inlet isothermal lobed jet on the mixing process with variable density have been studied numerically. For the same area exit geometries (axisymmetric and asymmetric), a qualitative comparison of the numerical results with experimental data have been presented. All these indicated the better mixing enhancement performance of a lobed nozzle over asymmetric and axisymmetric nozzles respectively.
\end{abstract}

Key words: Lobed jet / turbulent / variable density / streamwise vortex / RSM model

Résumé - Simulation numérique d'un jet turbulent lobé à masse volumique variable. Des simulations numériques de jets turbulents et isothermes issus de deux buses lobées sont présentés dans le présent article. Ces études numériques ont été effectuées en utilisant les équations de Navier-Stokes moyennées au sens de Favre, en utilisant aussi un modèle de turbulence de second ordre $(R S M)$. Le maillage choisi est structuré. La validation du modèle de calcul a été faite uniquement avec la première buse pour la même configuration. Cette comparaison montre un accord raisonnable, principalement, en termes de vitesse axiale moyenne, fluctuation axiale moyenne et la vorticité moyenne longitudinale. Dans la deuxième partie, les effets d'un jet lobé turbulent et isotherme à densité variable sur le processus de mélange ont été étudiés numériquement. Pour des surfaces de sortie équivalentes (axisymétriques et asymétriques), une comparaison qualitative des résultats numériques avec les données expérimentales est présentée. La buse lobée présente un meilleur mélange par rapport aux buses asymétrique et axisymétrique respectivement.

Mots clés : Jet lobé / masse volumique variable / vortex longitudinal / modèle RSM

\section{Introduction}

The physics of turbulent jet is of considerable interest from both fundamental and practical aspects. For fundamental aspect, it has been widely suggested that mixing process is intimately connected with transient of turbulence. Furthermore, the nozzle geometry and flow initial perturbations have profound influence on its generation and transition [1]. For practical aspect, the mixing process governs jet noise level of the airplane and vehicles, spread of pollutant at industrial sites and mixing rate in the combustion chambers. The turbulent jets with variable density emerging in a moving and confined atmosphere

\footnotetext{
a Corresponding author: boulenouar_m@yahoo.fr
}

are a complex physical problem. The coupling between the heat release due to chemical reactions and the mixing processes, the entrainment and recirculation of gases complicate the behavior of these jets. These flows are encountered in many engineering flows, such as: combustion, cooling in the turbo-machine engines by lateral injection, strongly heated jets at engine exit, plasma guns, heavy and accidental light gas ejection in the atmosphere, etc. Axisymmetric turbulent jets with variable density have been studied by different authors [2-8]. The development of the turbulent jet with variable density is very sensitive to the inlet conditions, particularly the injection ratio [9] and the direction of the co-flowing [10] and the geometry of the nozzle [11-14], which is the main object 


\section{Nomenclature}

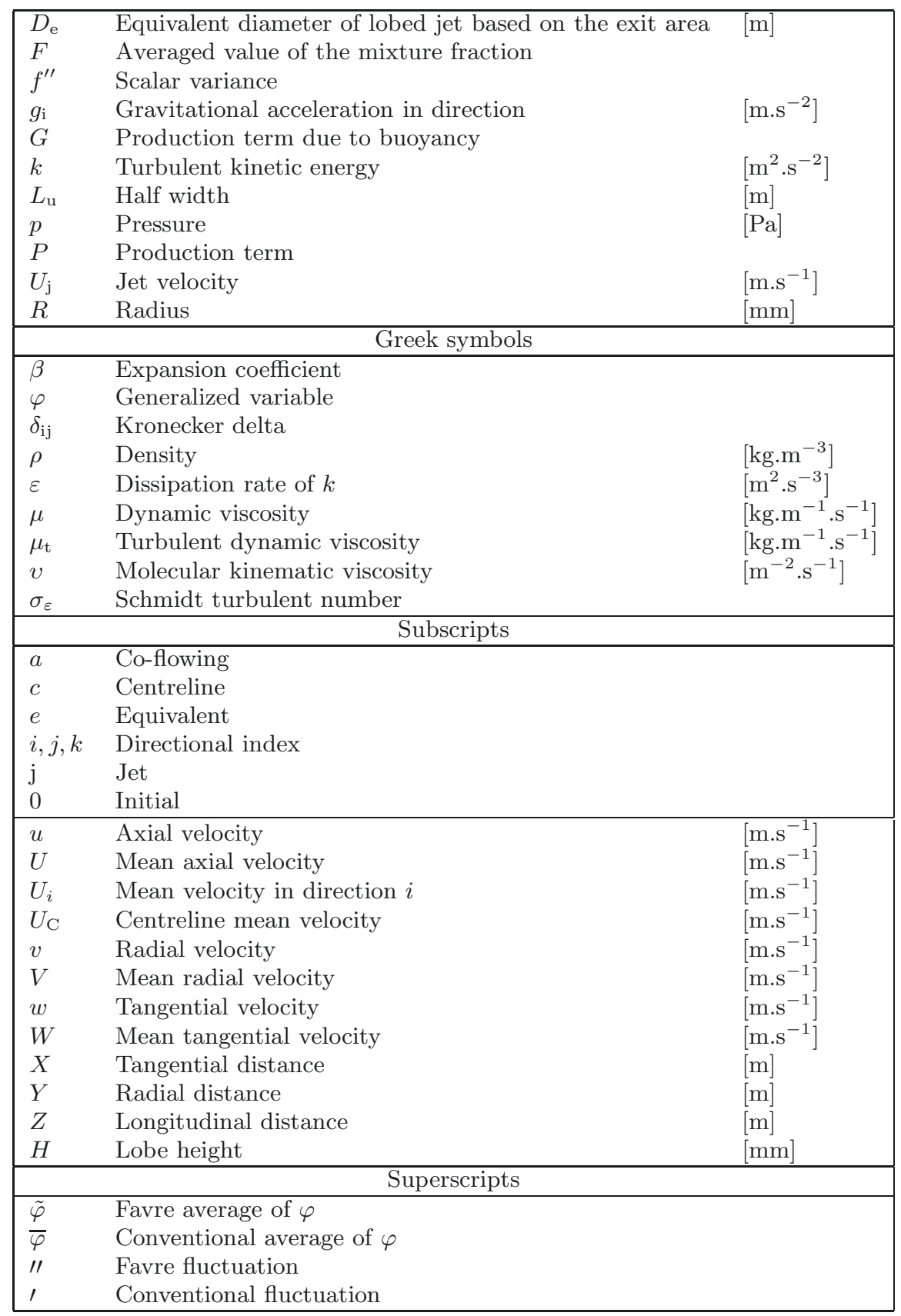

of the present study. Simulations of the turbulent jets employing a standard $\mathrm{k}-\varepsilon$ turbulence model have been performed by $[15,16]$. Their studies don't appear to be sufficient for capturing the mixing in these flows. Model predictions reveal that a very small degree of anisotropy is predicted throughout the flow field in comparison with experimental results. In the study of [17] a first and secondorder turbulence models have been used to investigate variable density effects in axisymmetric isothermal turbulent jet. The computed values show that only the secondorder model predicts correctly turbulence intensities. The authors of [18] have studied the jets with rectangular cross-section using the large eddy simulation (LES) and direct numerical simulation $(D N S)$. The phenomenon of axis-switching is observed to be dependent on instability waves present in the inlet boundary layers, and could be induced in both laminar and turbulent jets. In reference [19], the authors have presented numerical simulations of an incompressible jet mixing flow from a circular lobed nozzle and have validated by experimental measurements of the same configuration. The numerical simulation has been conducted using four two-equation turbulence models. All models over predict the magnitude of the turbulent kinetic energy. The author of [20] has 


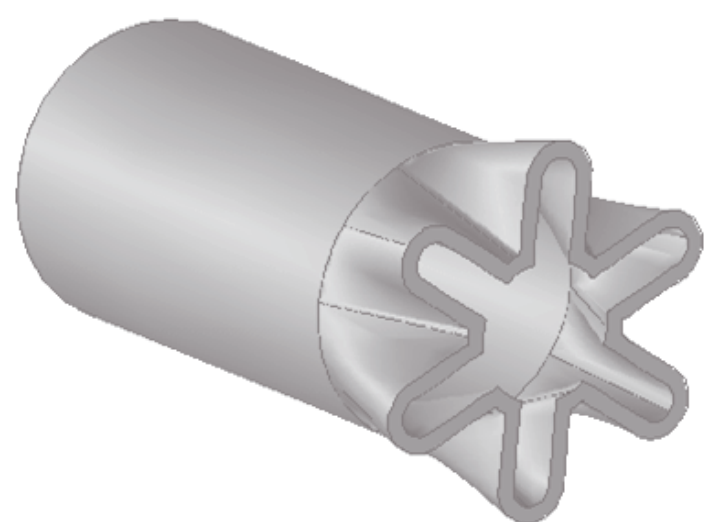

Fig. 1. Lobed nozzle.

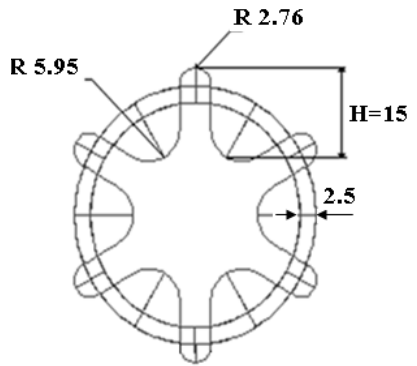

(a)

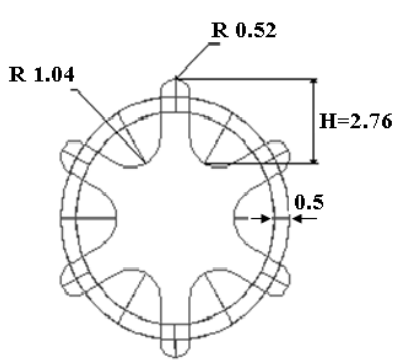

(b)
Fig. 2. View of the lobed geometries in the $(X Y)$ exit plane.

studied the influence of nozzle geometry on a turbulent binary gas mixing asymmetric jets using a second-order Reynolds Stress Model. The obtained results agree reasonably with experimental results. The main objective of the present study is to provide the quantitative information on the process mixing of the lobed nozzle by exploiting computational approach. The Reynolds stresses are modelled using the Reynolds Stress Model ( $R S M)$. Numerical simulations are proposed for two lobed nozzles ( $D_{\mathrm{e}}=40 \mathrm{~mm}$ and $D_{\mathrm{e}}=7 \mathrm{~mm}$ ) (see Fig. 1). The first part permits only to valid the numerical results with experimental data published in reference $[1,21]$. The second part provides detailed information on the lobed jet flow with variable density. The numerical results are compared with experimental measurements in reference [11].

\section{Geometries and flow conditions}

The first lobed nozzle is built up from a circular section tube of $D_{\mathrm{e}}=40 \mathrm{~mm}$ and $76.5 \mathrm{~mm}$ length. This straight tube is connected to a shorter lobed geometry having the same characteristics as indicated in the experimental study of [1]. The thickness of the wall at the exit plane is $2.5 \mathrm{~mm}$ (see Fig. 2a). The domain extends 10 equivalent diameters downstream of the exitplane and 4 equivalent diameters in transverse plane. The fluid medium is air. The incompressible jet mixing flow is injected with an initial volumetric flow rate $Q_{0}=4.7 \times 10^{-3} \mathrm{~m}^{3} \cdot \mathrm{s}^{-3}$. At the inlet, the turbulence intensities are estimated to be $1.1 \%$. The second lobed nozzle is built up from a circular section tube of $D_{\mathrm{e}}=7 \mathrm{~mm}$ diameter and $11 \mathrm{~mm}$ length. The thickness of the wall at the exit plane is $0.5 \mathrm{~mm}$ (see Fig. 2b). The jet is injected at atmospheric pressure with an inlet velocity $U_{\mathrm{j}}=40 \mathrm{~m} . \mathrm{s}^{-1}$. The jet is considered slightly confined and the co-flowing is considered cylindrical with a diameter $D_{\mathrm{a}}=300 \mathrm{~mm}$ and a length $L_{\mathrm{a}}=2000 \mathrm{~mm}$. The co-flowing (air) is injected with a velocity $U_{\mathrm{a}}=0.36 \mathrm{~m} . \mathrm{s}^{-1}$ at the same pressure condition as the jet. Lobed nozzles have the same inner and outer lobe penetration angles $\left(22^{\circ}, 14^{\circ}\right)$. Jets exit planes have six lobes with parallel sides and six troughs of sinusoidal shape.

\section{Numerical formulation}

\subsection{Governing equations}

In the mathematical description of the conservation equations, all variables, except the pressure and the density, which are always computed according to Reynolds average, are Favre average (mass-weighted). This quantity is defined as:

$$
\tilde{\varphi}=\frac{\overline{\rho \varphi}}{\bar{\rho}}
$$

- Average continuity equation

$$
\frac{\partial\left(\bar{\rho} \tilde{U}_{i}\right)}{\partial x_{j}}=0
$$

- Average momentum conservation equation

$$
\begin{aligned}
\frac{\partial\left(\bar{\rho} \tilde{U}_{i} \tilde{U}_{j}\right)}{\partial x_{j}} & =\bar{\rho} g_{i}-\frac{\partial \bar{p}}{\partial x_{i}}-\frac{\partial\left(\overline{\left.\rho u_{i}^{\prime \prime} u_{j}^{\prime \prime}\right)}\right.}{\partial x_{j}} \\
+ & {\left[\frac{\partial}{\partial x_{j}} \bar{\mu}\left(\frac{\partial \tilde{U}_{i}}{\partial x_{j}}+\frac{\partial \tilde{U}_{j}}{\partial x_{i}}\right)-\frac{2}{3} \bar{\mu} \frac{\partial \tilde{U}_{k}}{\partial x_{k}} \delta_{i j}\right] }
\end{aligned}
$$

- Average mixture fraction conservation equation

$$
\begin{aligned}
\frac{\partial\left(\bar{\rho} \tilde{F} \tilde{U}_{j}\right)}{\partial x_{j}} & =-\frac{\partial \bar{d}_{j}^{k}}{\partial x_{j}}-\frac{\partial}{\partial x_{j}} \overline{\left(\rho f^{\prime \prime} u_{j}^{\prime \prime}\right)} \\
\overline{\mathrm{d}}_{j}^{k} & =-\frac{\lambda}{C_{p}} \frac{\partial \tilde{F}}{\mathrm{~d} x_{j}}
\end{aligned}
$$

The mean density can be obtained from the mean mixture fraction using the equation of state. With constant pressure, this leads to:

$$
\frac{1}{\bar{\rho}}=\frac{F}{\rho_{j}}+\frac{1-F}{\rho_{a}}
$$

\subsection{Reynolds Stress Model (RSM)}

The Reynolds stresses $\rho \overline{u^{\prime \prime} u^{\prime \prime}}, \rho \overline{v^{\prime \prime} v^{\prime \prime}}$ and $\overline{\rho w^{\prime \prime} w^{\prime \prime}}$ are defined as:

$$
\begin{array}{r}
\frac{\partial}{\partial x_{k}}\left(\tilde{U}_{k} \overline{\rho u_{i}^{\prime \prime} u_{j}^{\prime \prime}}\right)=P_{i j}+G_{i j}+D_{i j}+p^{\prime} \overline{\left(\frac{\partial u_{i}^{\prime \prime}}{\partial x_{j}}+\frac{\partial u_{j}^{\prime \prime}}{\partial x_{i}}\right)} \\
-\frac{2}{3} \bar{\rho} \varepsilon \delta_{i j}
\end{array}
$$




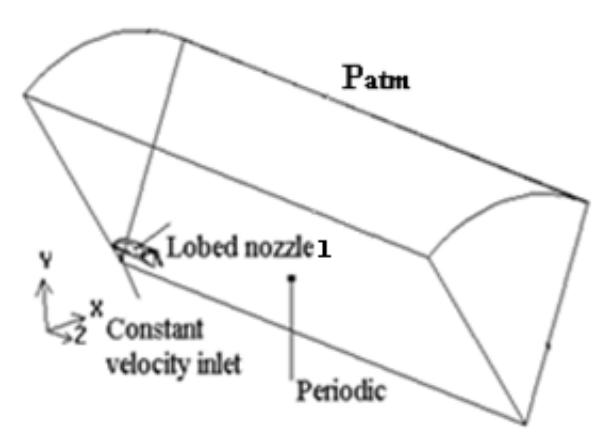

(a)

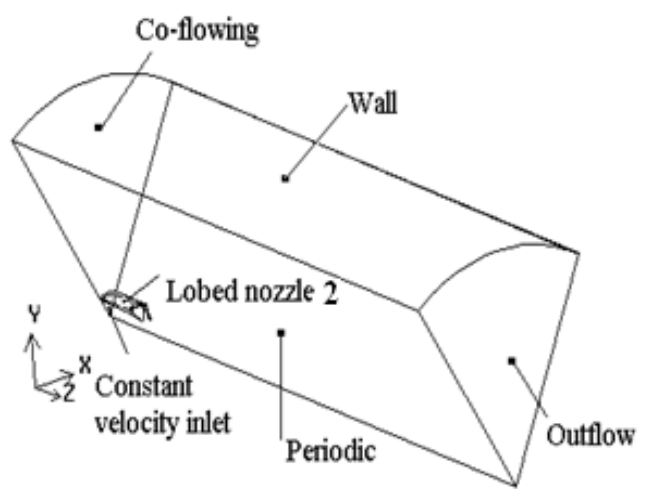

(b)

Fig. 3. Computational domains and boundary conditions.

where the assumption of the isotropy for the smallest scales has been assumed.

The term $P_{i j}$ is the production term due to the mean strain and is defined as:

$$
P_{i j}=-\left(\overline{\rho u_{i}^{\prime \prime} u_{k}^{\prime \prime}}\right) \frac{\partial \tilde{U}_{\mathrm{j}}}{\partial x_{k}}-\overline{\left(\rho u_{j}^{\prime \prime} u_{k}^{\prime \prime}\right)} \frac{\partial \tilde{U}_{i}}{\partial x_{k}}
$$

The term $G_{i j}$ is the production due to the buoyancy effects and is modeled as:

$$
G_{i j}=-\beta\left(g_{i} \overline{\rho u_{j}^{\prime \prime} f^{\prime \prime}}+g_{j} \overline{\rho u_{i}^{\prime \prime} f^{\prime \prime}}\right)
$$

The diffusion term $D_{i j}$ is modeled as:

$$
D_{i j}=C_{s} \frac{\partial}{\partial x_{k}}\left[\left(\frac{k}{\varepsilon}\left(\rho u_{k}^{\prime \prime} u_{l}^{\prime \prime}\right)+\bar{\rho} \delta_{k l} \nu\right) \frac{\partial\left(\overline{\rho u_{i}^{\prime \prime} u_{j}^{\prime \prime}}\right)}{\partial x_{j}}\right]
$$

Here, the turbulent kinetic energy is defined as:

$$
k=\frac{1}{2} \overline{u_{i}^{\prime \prime} u_{i}^{\prime \prime}}
$$

The dissipation rate equation is exactly the same as in the standard $\mathrm{k}-\varepsilon$ model and has the form:

$$
\begin{aligned}
\frac{\partial}{\partial x_{k}}\left(\bar{\rho} \tilde{U}_{k} \varepsilon\right)=\frac{\varepsilon}{k}\left(C_{\varepsilon 1} P\right. & \left.-C_{\varepsilon 2} \bar{\rho} \varepsilon\right) \\
& +\frac{\partial}{\partial x_{k}}\left[\left(\mu+\frac{\mu_{t}}{\sigma_{\varepsilon}}\right) \frac{\partial \varepsilon}{\partial x_{k}}\right]
\end{aligned}
$$

One can find more details concerning modelling of the Reynolds stress equations and their constants in reference [17].

\section{Boundary conditions}

For all calculations, because of symmetry, only the sixth of the physical field is considered as computational domains. For lobed nozzle 1, constant static pressure is considered across the outer domain extends with rotationally periodic boundary conditions imposed on each side (see Fig. 3a).
For the lobed nozzle 2, the boundary conditions are written as follows:

- At the inlet and in order to overcome as much as possible the influence of the jet and the co-flowing emissions, the velocity, the Reynolds stresses and the turbulent kinetic energy profiles were calculated by extrapolating at $Z / D_{\mathrm{e}}=0.3[11]$. The lateral and transverse velocities and scalar variance are zero. The mixture fraction is one at inlet jet and zero at inlet coflowing. It should be noted, that no experimental data are available on the dissipation rate of the turbulent kinetic energy at the emission section. At the emission section, the length scale $L_{\mathrm{m}}$ and the dissipation rate $\varepsilon$ are estimated as follows:

$$
\begin{aligned}
L_{\mathrm{m}} & =0.007 D_{\mathrm{e}} \\
\varepsilon & =C_{\mu} \frac{k^{\frac{3}{2}}}{L_{\mathrm{m}}}
\end{aligned}
$$

- On the periodic planes, all variables have a null partial normal derivative, with the exception of the lateral and the transverse velocities are zero.

- Wall is taken into account by using wall function that imposes a value for each function in the first grid division, at a distance from the wall located in the logarithmic region of the velocity profile.

- At the outflow boundary, the gradients of dependent variables in the axial direction are set to zero.

\section{Numerical method}

Lobed nozzles and domains are meshed using the software Gambit 2.3.16. Three grid sizes have been tested for the grid independency of the solution for the lobed nozzle 1 (see Figs. 4 and 5) and other three grid sizes for lobed nozzle 2 (see Figs. 6, 7) with an expansion factor equal to 2 (see Tab. 1 and 2). The grids were selected when no are significant differences between the results on the Reynolds stress and dissipation rate. The final meshes are the grids 882883 and 821240 cells. The domains are meshed using structured cells (see Fig. 8). The equations describing 


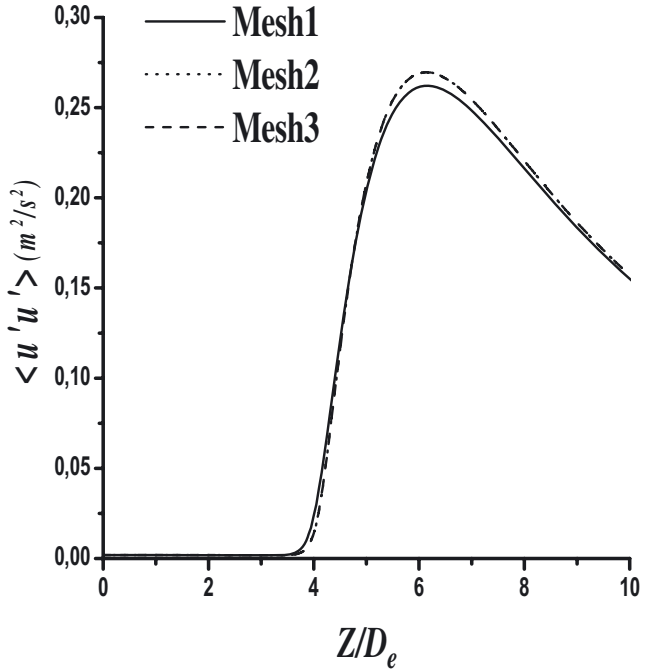

Fig. 4. Centreline evolution of the Reynolds stress $<u^{\prime} u^{\prime}>$ for different meshes, $D_{\mathrm{e}}=40 \mathrm{~mm}$.

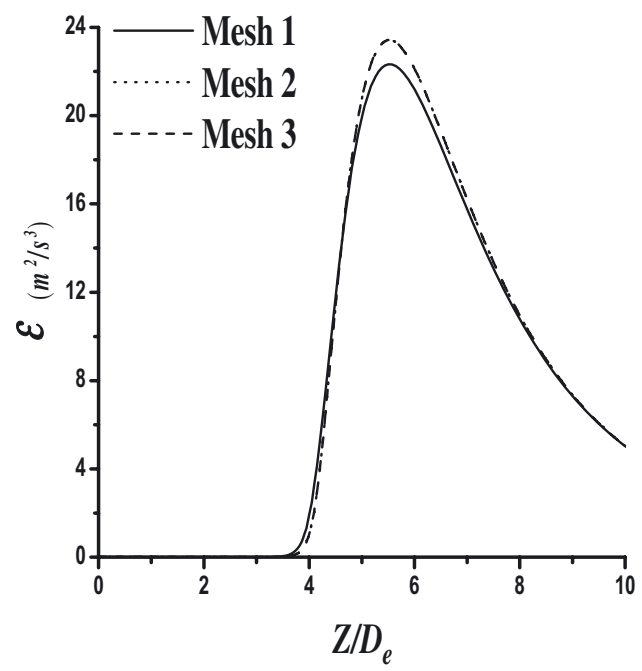

Fig. 5. Centreline evolution of the dissipation rate $\varepsilon$ for different meshes, $D_{e}=40 \mathrm{~mm}$.

Table 1. Meshes tested for lobed nozzle 1.

\begin{tabular}{ccc}
\hline & $\begin{array}{c}\text { Number of } \\
\text { cells }\end{array}$ & $\begin{array}{c}\text { Number of nodes } \\
\text { on }(x-y-z)\end{array}$ \\
\hline Mesh 1 & 440956 & $28-33-275$ \\
Mesh 2 & 882883 & $30-55-423$ \\
Mesh 3 & 1771782 & $40-66-554$ \\
\hline
\end{tabular}

a turbulent flow are of elliptic convection-diffusion. The SIMPLE algorithm is used for pressure-velocity coupling. The second order upwind discretization scheme was employed in order to achieve better convergence. The convergence criterion required for the computed residuals is less than $10^{-6}$ for all equations. The numerical analysis is performed using a finite volume based solver Fluent 6.3.26.

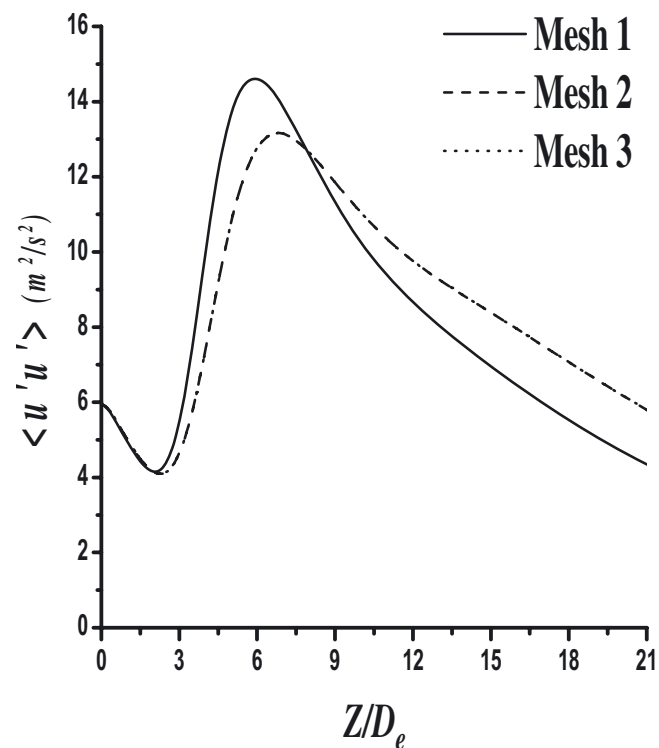

Fig. 6. Centreline evolution of the Reynolds stress $\left\langle u^{\prime} u^{\prime}\right\rangle$ for different meshes, $D_{\mathrm{e}}=7 \mathrm{~mm}$.

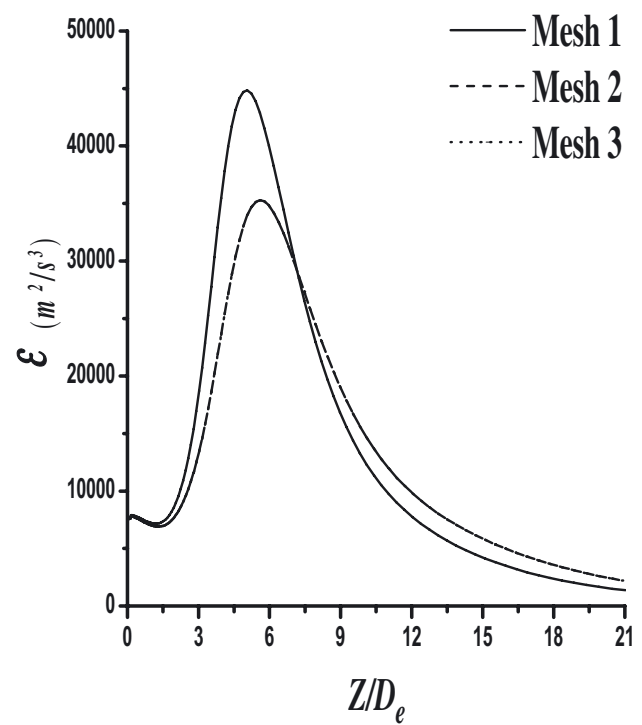

Fig. 7. Centreline evolution of the dissipation rate $\varepsilon$ for different meshes, $D_{\mathrm{e}}=7 \mathrm{~mm}$.

Table 2. Meshes tested for lobed nozzle 2.

\begin{tabular}{ccc}
\hline & $\begin{array}{c}\text { Number of } \\
\text { cells }\end{array}$ & $\begin{array}{c}\text { Number of nodes } \\
\text { on }(x-y-z)\end{array}$ \\
\hline Mesh 1 & 409888 & $20-40-410$ \\
Mesh 2 & 821240 & $24-49-590$ \\
Mesh 3 & 1646116 & $32-62-719$ \\
\hline
\end{tabular}

\section{Results and discussion}

\subsection{Numerical validation of the results}

It should be noted that the entire simulated flow field is created by duplicating and periodically rotating the computational domains (periodic on $60^{\circ}$ intervals) 


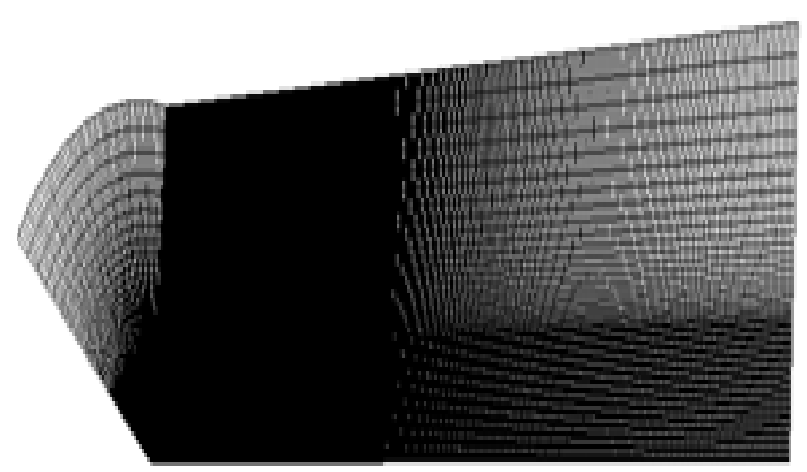

Fig. 8. Structured mesh.

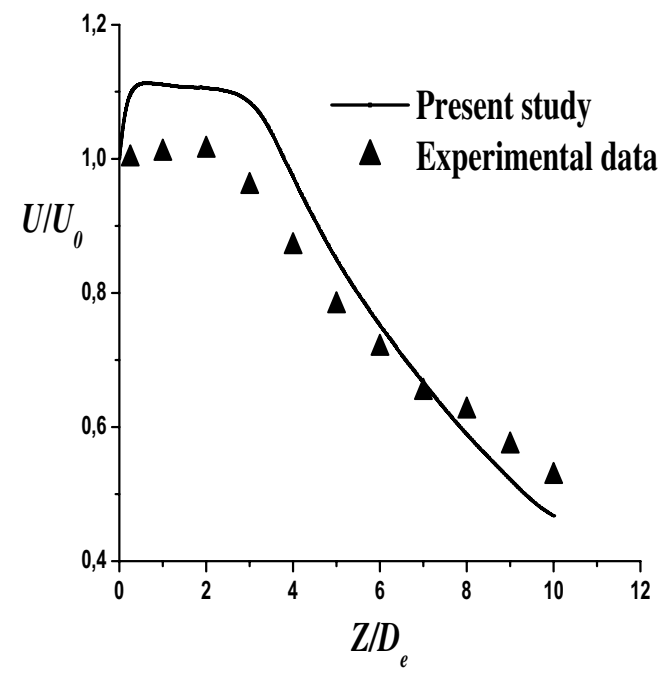

Fig. 9. Centreline evolution of the normalized axial velocity for air-air.

through a full $360^{\circ}$. The evolution of the centreline axial velocity normalized by the centreline velocity at jet exit $U_{0}$ is shown in Figure 9. It is found that the turbulent model predicts $10 \%$ velocity increase at the jet exit, while the experimental results show only $2 \%$ velocity increase. The length of the potential core of the simulated flow appears to agree reasonably well with experimental data $\left(3 D_{\mathrm{e}}\right.$ to compare to $2 D_{\mathrm{e}}$ in reality). After this region, a satisfactory agreement is obtained on the velocity levels since the difference between the simulation and the reference is less then $5 \%$. Figure 10 shows the streamwise evolution of the normalized longitudinal volumetric flow rate, where $Q$ represents the volumetric rate for lobed jet calculated by (15) and $Q_{0}$ the initial volumetric flow rate.

$$
Q=\int r U \mathrm{~d} r
$$

Only the values larger than $0.2 \mathrm{~m} . \mathrm{s}^{-1}$ were taken into account [1]. This value is considered by [1] as the criterion defining the extinction of the flow from the point of view of the thermal and draft comfort of the occupants. The numerical flow rate evolution law is close to the experimental one with a relative maximum deviation of $7 \%$ at $Z=10 D_{\mathrm{e}}$. From the position $Z=2 D_{\mathrm{e}}$, the curves are

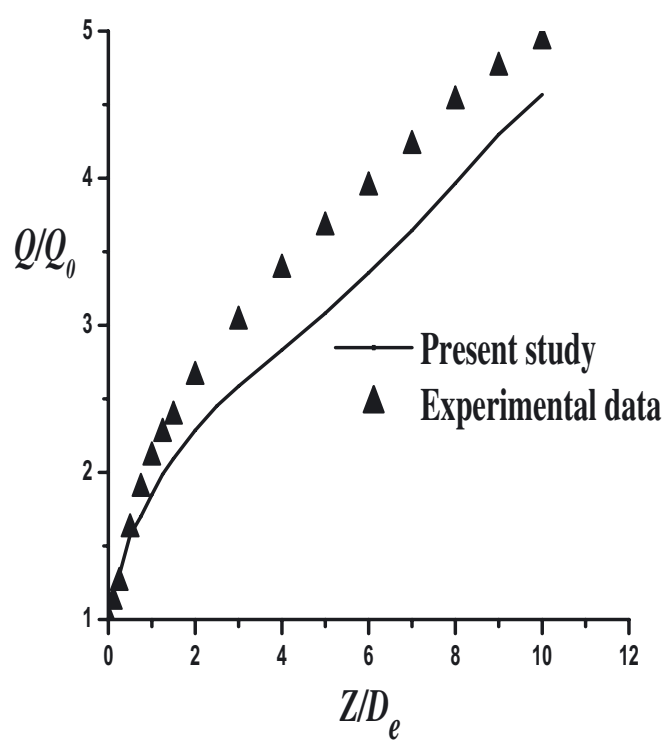

Fig. 10. Longitudinal evolution of the normalized volumetric flow rate for air-air.

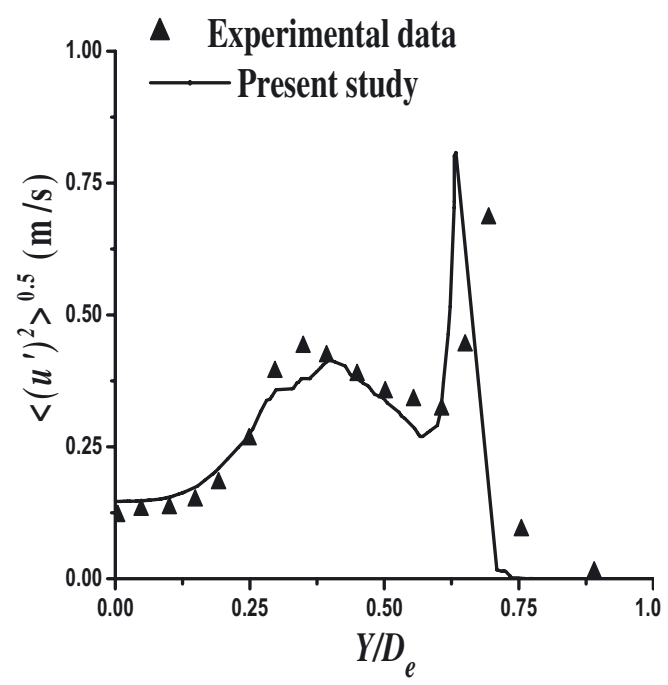

Fig. 11. Radial evolution of the axial velocity fluctuation at $Z / D_{\mathrm{e}}=0.25$ for air-air.

linear. On the exit radial distribution of the mean fluctuating streamwise velocity is shown in Figure 11, the two peaks of the curve at $Z \approx 0.4 D_{\mathrm{e}}$ and $Z \approx 0.6 D_{\mathrm{e}}$ respectively, are well predicted by the model. These peaks are related to areas of high velocity gradients due to connection between the jet core flow and the lobe flow and between the periphery of the jet flow and the zero speed in the ambient air respectively. Figures $12 \mathrm{a}$ and $\mathrm{b}$ give at the streamwise position $Z=0.25 D_{\mathrm{e}}$, the normalized streamwise vorticity distribution of the present numerical simulation and of the experimental data respectively. The normalized streamwise vorticity $\varpi_{\mathrm{Z}}$ is defined as follows:

$$
\varpi_{\mathrm{Z}}=\frac{D_{\mathrm{e}}}{U_{0}}\left(\frac{\partial V}{\partial Y}-\frac{\partial U}{\partial X}\right)
$$




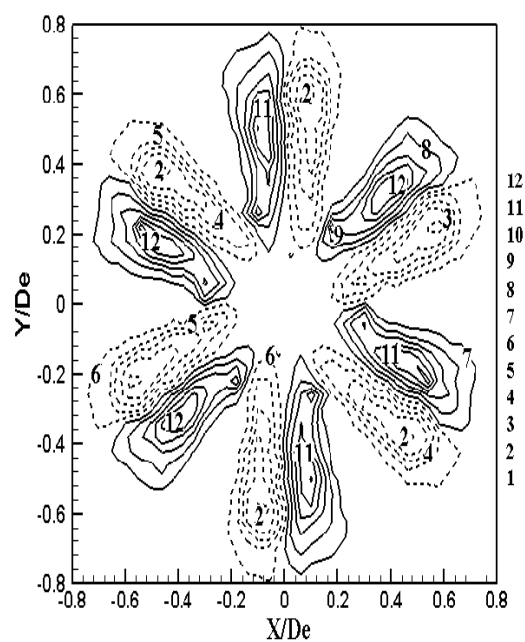

(a)

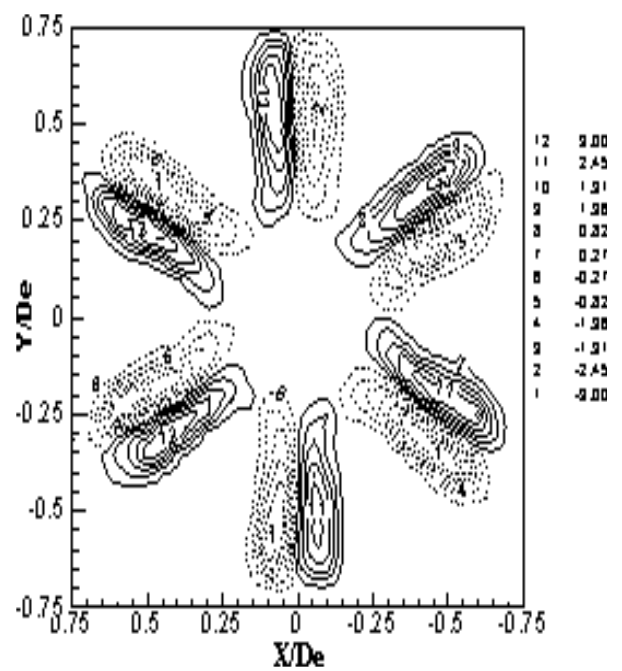

(b)

Fig. 12. (a) Normalized mean longitudinal vorticity distribution (present study) for air-air. (b) Normalized mean longitudinal vorticity distribution (experimental data) for air-air.

The simulated transverse field agrees reasonably well with the experimental one. In fact, the six pairs of counter rotating large scale streamwise structures generated by the geometry of the lobed diffuser are predicted by the model. Each structure corresponds to the shear generated by two inverse transverse flows: outward flow due to the outer lobe penetration angle and inward flow due to the inner lobe through penetration angle. It is interesting to note that the maximal level of $\varpi_{Z}$ is equal to the experiment maximal value.

\subsection{Analysis of the characteristics of the lobed jet flow}

Figure 13 shows the evolution of the normalized axial velocity on the radial axis at different cross sections. At $Z / D_{\mathrm{e}}=1.5$, the initial development of a thin shear layer is observed. Up to this location, the velocity in the vicinity of the jet centreline remains constant and is equal to the inlet jet prescribed velocity. The potential core of the lobed jet is located before $Z / D_{\mathrm{e}}=6$ and the jet width is larger than that at the exit. The axial velocity at $Z / D_{\mathrm{e}}=15$ has attained a similar distribution as in elliptical jet (see Ref. [20]). The velocity half width $L_{\mathrm{u}}$ is defined in a cross section as the radial position where the axial velocity is equal to $U_{c} / 2$. Figure 14 represents the growth of the velocity half width for a lobed jet 2 , with $\mathrm{CO}_{2}$-air, in the radial and transversal axis directions. It is shown a difference of the jet growth rate according to these two directions. This is due to the three-dimensional effects. It is observed that the curves in both axis directions meet at $Z / D_{\mathrm{e}} 6$ coincide and remain approached beyond this value. The jet appears to be nearly rounded, although it is lobed at nozzle exit. The streamwise velocity half width changes showing that the spreading of the lobed jet in the self-preserving region is not perfectly linear. The normalized longitudinal velocity distributions

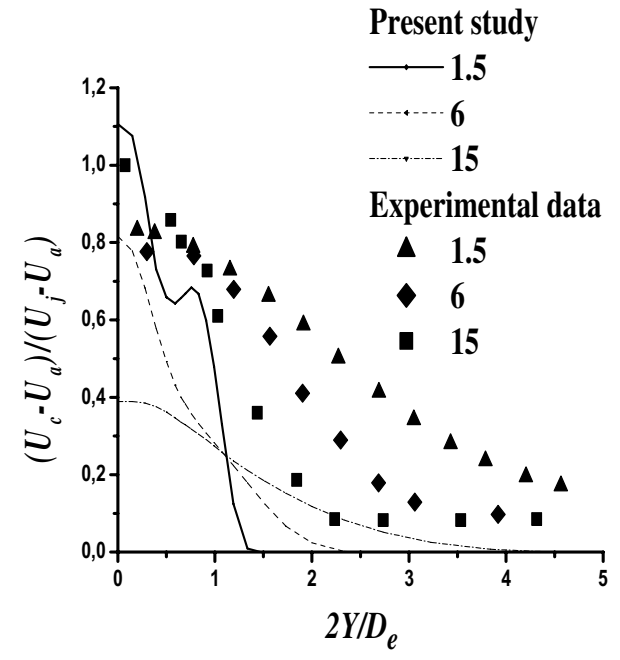

Fig. 13. Radial evolution of the normalized axial velocity for $\mathrm{CO}_{2}$-air.

are showed in Figure 15 at different sections. It is noted that the lobe signature, extremely intense at $Z / D_{\mathrm{e}}=0.2$, tends to disappear at $Z / D_{\mathrm{e}}=6$ as distinguished in [14] at $Z / D_{\mathrm{e}}=3$. For $Z / D_{\mathrm{e}}>0.2$, the jet size increases similarly along the radial and transversal axis. Further downstream at $Z / D_{\mathrm{e}}=9$, contour plots become almost circular.

\subsection{Comparison of the numerical results with experimental measurements using different geometries}

At the same conditions of jet flow, the lengths of potential core and decay of longitudinal velocities in the initial regions are considered as the indicators of the level mixing [22]. In order to analyze the mixing 


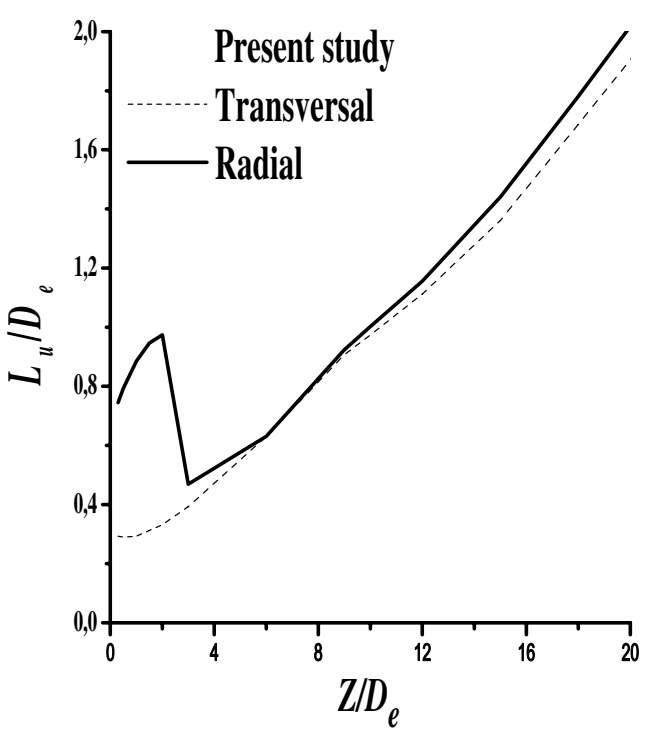

Fig. 14. Longitudinal evolution of the jet half width with different geometries for $\mathrm{CO}_{2}$-air.

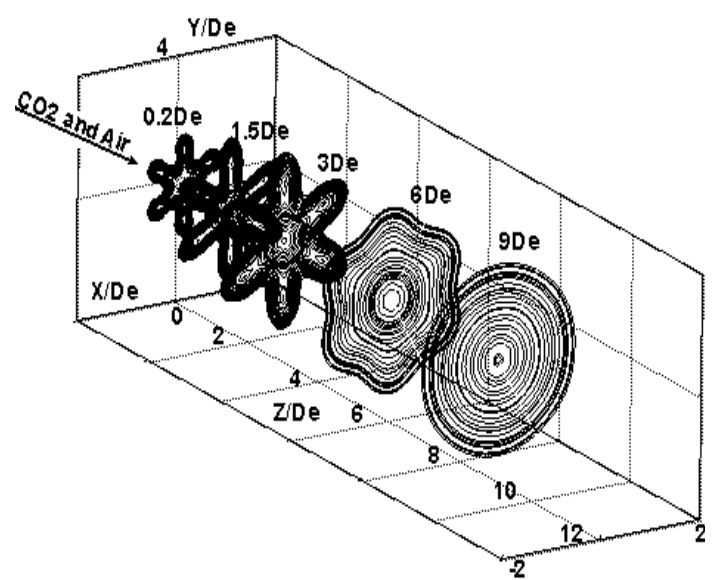

Fig. 15. Normalized axial velocity contours at $Z / D_{\mathrm{e}}=0.2$, 1.5, 3, 6 and 9 for $\mathrm{CO}_{2}$-air.

performance for the lobed jet 2, it is proposed in Figure 16 a comparison of the centerline normalized inverse velocity $\left(U_{\mathrm{j}}-U_{\mathrm{a}}\right) /\left(U_{c}-U_{\mathrm{a}}\right)$ between circular, elliptical and rectangular jets experimental measurements of [11] and lobed jet 2 numerical results for $\mathrm{CO}_{2}$-air. The normalized inverse axial velocity is defined as:

$$
\frac{U_{\mathrm{j}}-U_{\mathrm{a}}}{U_{\mathrm{c}}-U_{\mathrm{a}}}=\frac{1}{K_{\mathrm{V}}}\left(\frac{D_{\mathrm{e}}}{Z-Z_{0}}\right)
$$

In Figure 16, the numerical results show the existence of two distinct regions. The first region is near jet exit where the velocity takes an increase of $10 \%$ with respect to $U_{\mathrm{j}}$ until $Z / D_{\mathrm{e}}=0.6$ while the experimental measurements [11] are not showed increase in this region. At the exit section of the lobed nozzle, the increases in the longitudinal velocities are the effect of the particular form of the lobe. In their experimental study, the authors of the reference [1] have showed the role played by the deflection angles of the lobed nozzle in the mixing enhancement.

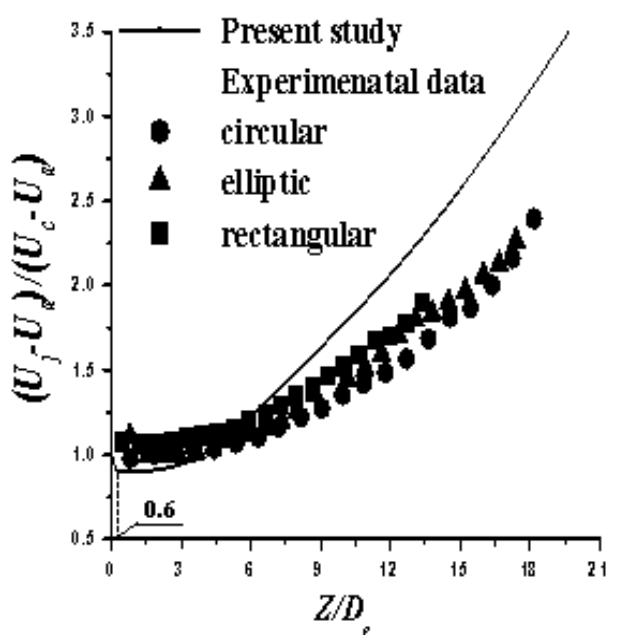

Fig. 16. Centreline evolution of the normalized inverse axial velocity for $\mathrm{CO}_{2}$-air.

Table 3. Values of the potential core length for $\mathrm{CO}_{2}$-air.

\begin{tabular}{cc}
\hline Geometries & \\
\hline Circular (Ref. [11]) & 17.1 \\
Rectangular (Ref. [11]) & 9.6 \\
Elliptic (Ref. [11]) & 7.5 \\
Lobed (present study) & 6.58 \\
\hline
\end{tabular}

It has been suggested in [21] that the lobed nozzle is an extraordinary mixing device. The exploration of the initial region revealed the existence of a pair of large scale streamwise vortices at every lobe peak which dominate spreading and mixing enhancement phenomena. For $Z / D_{\mathrm{e}}>0.6$, the velocity remains constant but less than $U_{\mathrm{j}}$. This is the region of the potential core. Beyond the potential core, differences between curves increase. At the position $Z / D_{\mathrm{e}}=12.5$, it is noted a decrease in the average longitudinal velocity of the lobed jet to about $16.5 \%$ compared to the rectangular jet, $20.2 \%$ for the elliptical, $28.5 \%$ for the circular. Figure 17 shows a comparison of the velocity half width for different geometries and for the $\mathrm{CO}_{2}$-air. It is found that the lobed jet 2 develops more strongly than other jets, particularly with the circular jet. Figure 18 shows a comparison between the computed normalized fluctuating velocity for different geometries. In spite of the variations which are due to the strong dependence of the geometries conditions, the numerical and experimental results present a similar longitudinal evolution. From the Figure 19, it can be seen that the centreline evolutions of the turbulent kinetic energy show stronger values for a lobed jet than for the configurations of [11] in the near field. The origin of these differences is due to particular form of the lobed nozzle 2. The author of [11] assimilates the position of the maximum axial kinetic energy to the potential core length (Tab. 3). This length is shorter in the case of the lobed jet than in the rectangular, elliptic and circular jets. The largest length is obtained with the circular jet. 


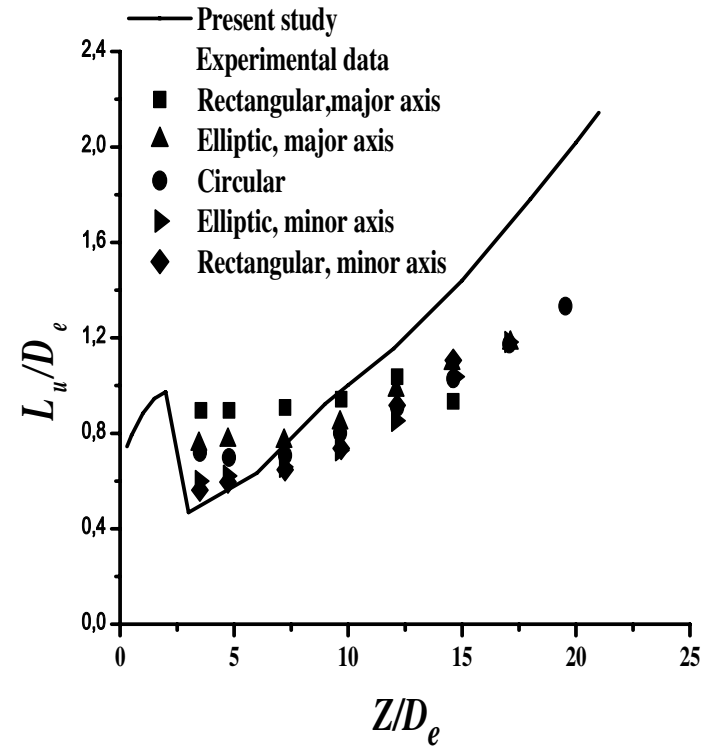

Fig. 17. Longitudinal evolution of the jet half width for $\mathrm{CO}_{2-}$ air.

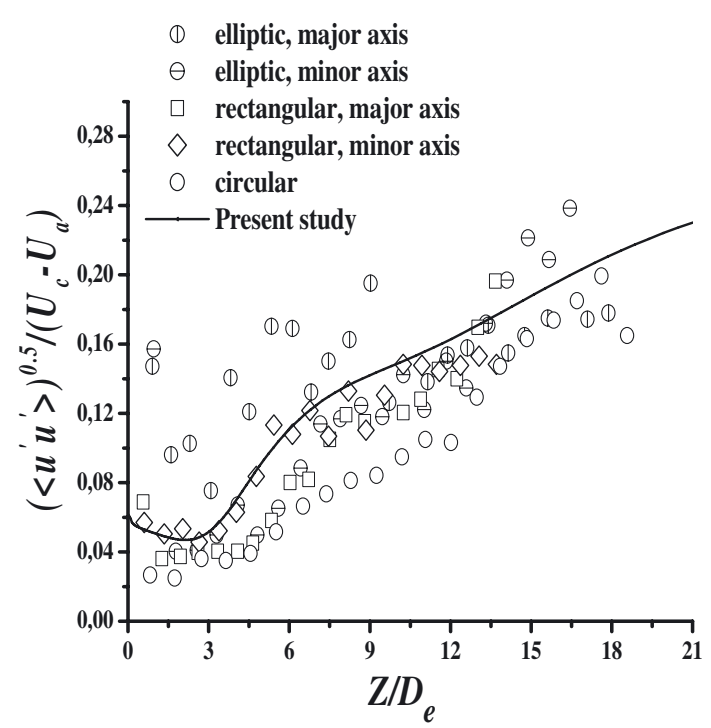

Fig. 18. Centreline evolution of the normalized axial velocity fluctuation for $\mathrm{CO}_{2}$-air.

\subsection{Comparison of the lobed jet numerical results to numerical ones using elliptic geometry for different densities}

The effect of the density on the inverse normalized centreline velocity and inverse mixture fraction are showed in Figures 20 and 21. For the region $Z / D_{\mathrm{e}}>4$, the evolution of these quantities correspond to same behavior that is showed in the reference [20]. Furthermore, it is noted that the reduction in the jet density results in an increase of the decay rate of the normalized centreline velocity and the mixture fraction. Also these results indicated the better mixing enhancement performance of a lobed jet over an elliptic jet.

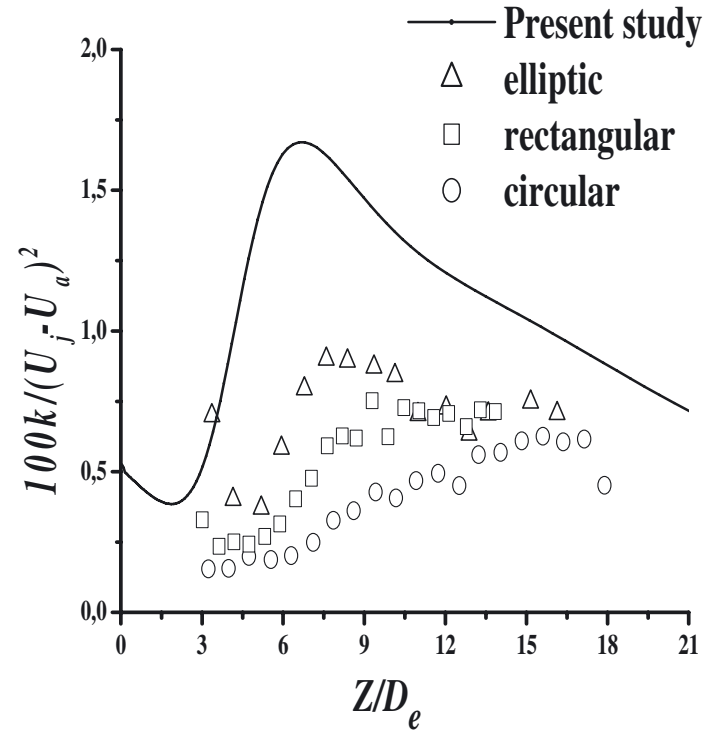

Fig. 19. Centreline evolution of the normalized turbulent kinetic energy for $\mathrm{CO}_{2}$-air.

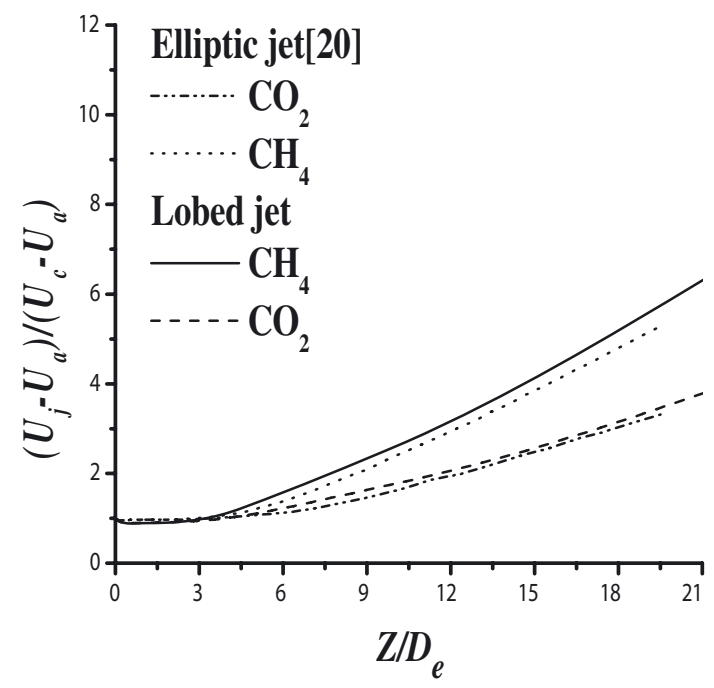

Fig. 20. Centreline evolution of the normalized inverse longitudinal velocity for different geometries and mixtures.

\section{Conclusion}

Numerical simulations of circular lobed turbulent jets mixing flow by solving a three-dimensional form of the Favre-Reynolds averaged Navier-Stokes equations with structured cells are proposed in the present study. Two lobed jets with different sizes and similar penetration angles have been studied. The numerical results of the first lobed 1 are validated by using measurement data in reference [1] for the same configuration. It is showed several features of the mixing process such as: centreline axial velocity, axial velocity fluctuation. The numerical results follow similar trend that the experimental data. However, the numerical model $(R S M)$ over predicts the maximum of the axial velocity fluctuation and its position. It is showed that the numerical results of the mean 


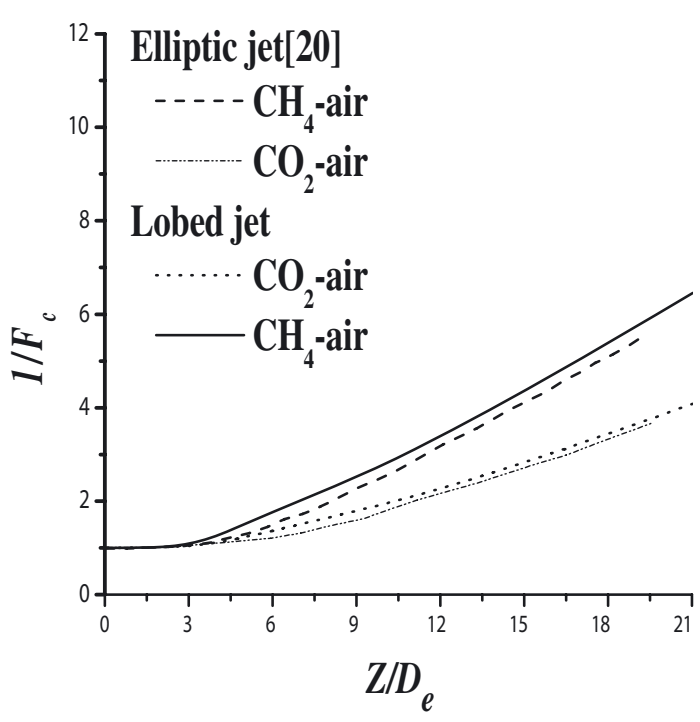

Fig. 21. Centreline evolution of the inverse mixture fraction for different geometries and mixtures.

streamwise vorticity distributions agree with the experimental measurements. Furthermore, jet's effects of the lobed nozzle 2 on an isothermal turbulent jet with coflowing, slightly confined, have been numerically investigated with different mixtures. All results indicated the better mixing enhancement performance of a lobed nozzle over asymmetric and axisymmetric nozzles respectively.

\section{References}

[1] I. Nastase, A. Meslem, Vortex dynamics and mass entrainment in turbulent lobed jets with and without lobe deflection angles, Exp. Fluids 48 (2010) 693-714

[2] R. So, J.H. Zhu, Some measurements in a binary gas jet, Exp. Fluids 9 (1990) 237-284

[3] F.C. Gouldin, R.W. Schefer, S.C. Johnson, W. Kollmann, Non-reacting turbulent mixing flows, Progr. Energ. Combust. Sci. 12 (1986) 257-303

[4] L. Fulachier, F. Anselmet, M. Amielh, Turbulent jets with variable density, PRC combustion in the rocket motors cryotechnic, CNRS/CNES/SEF, 1990

[5] N.R. Panchapakesan, J. Lumley, Turbulence measurements in axisymmetric jets of air and helium, Part 1 airjet, Part 2 helium-jet, Fluid Mech. (1993) 225-247

[6] A. Purwanto, Modélisation d'écoulements turbulentsbasse vitesse à forte variation de masse volumique, Thèse de doctorat, I. N. P de Toulouse, 1994
[7] L. Pietri, M. Amielh, F. Anselmet, Simultaneous measurements of temperature and velocity fluctuations in a highly heated jet combining a cold wire and laser Doppler, Int. J. Heat, Fluid Flow 21 (2000) 22-36

[8] M.H. Gazzah, M. Sassi, B. Sarh, I. Gökalp, Simulation numérique des jets turbulents subsoniques à masse volumique variable par le modèle $\mathrm{k}-\varepsilon$, Int. J. Therm. Sci. 41 (2002) 51-62

[9] H. Mhiri, Étude numérique des conditions d'émission sur un écoulement de type jet plan turbulent isotherme ou chauffé, Revue générale de thermique 38 (1999) 904-915

[10] B. Imine, Études numériques sur les jets turbulents à masse volumique variable, Thèse de doctorat d'état, Université M. Boudiaf Algérie, 2005

[11] B. Mesnier, Études sur le développement de jets turbulents à masse volumique variable à géométries axisymétrique et asymétrique, Thèse de doctorat, Université d'Orléans, 2001

[12] W.R. Quinn, On mixing in an elliptic turbulent free jet, Phys. Fluids 1 (1989) 1716-1722

[13] K.B.M.Q. Zaman, Spreading characteristics and thrust of jets from asymmetric nozzles, AIAA, 1996, 96-0200

[14] H. Hu, T. Kobayashi, T. Saga, N. Taniguchi, A study on a lobed jet mixing flow by using stereoscopic PIV tech, Phys. fluids 13 (2001) 3425-3441

[15] P. Koutmos, J.J. McGuirk, Turbofan Forced Mixer/Nozzle Temperature and Flow Field Modeling, Int. J. Heat Mass Transfer 32 (1989) 1141-1153

[16] H. Salman, G.J Page, J.J. McGuirk, Prediction of Lobed Mixer Vortical Structures with a k- $\varepsilon$ Turbulence Model, AIAA J. 41 (2003) 878-887

[17] J.P. Sanders, B. Sarh, I. Gokalp, Variable density effects in axisymmetric isothermal turbulent jet: a comparison between a first and a second order turbulence model, Int. J. Heat Mass Transfer 40 (1997) 823-842

[18] A.O. Demuren, R.V. Wilson, Streamwise vorticity generation in laminar and turbulent jets, NASA/CR 209517, ICASE Report 99-33, 1999, pp. 1-11

[19] J.C. Nathan, M. Parviz, H. Hu, Numerical simulation of the vortical structures in a lobed jet mixing flow, 43rd AIAA Aerospace Sciences Meeting and Exhibit, Reno Nevada, 2005-0635, pp. 1-13

[20] B. Imine, O. Imine, M. Abidat, A. Liazid, Study of nonreactive isothermal turbulent asymmetric jet with variable density, Comp. Mech. 385 (2006) 151-162

[21] I. Nastase, A. Meslem, K. Abed-Meraim, An experimental investigation on the asymmetric evolution of a lobed jet flow, Int. J. Dynamics Fluids 2 (2006) 73-97

[22] I. Nastase, Analyse des jets lobés en vue de leur intégration dans les unités terminales de diffusion d'air, Thèse, Université de La Rochelle, 2007 